Sharif University of Technology
Scientia Iranica
Transactions E: Industrial Engineering
w I I ENTw.scientiairanica.com

\title{
A class of multi-objective mathematical programming problems in a rough environment
}

\author{
A. Hamzehee ${ }^{a, c, *}$, M.A. Yaghoobi ${ }^{a}$ and M. Mashinchi ${ }^{b}$ \\ a. Department of Mathematics, Faculty of Mathematics and Computer, Shahid Bahonar University of Kerman, Kerman, Iran. \\ b. Department of Statistics, Faculty of Mathematics and Computer, Shahid Bahonar University of Kerman, Kerman, Iran. \\ c. Department of Mathematics, Faculty of Basic Science, Kerman Branch, Islamic Azad University, Kerman, Iran.
}

Received 13 August 2013; received in revised form 9 July 2014; accepted 17 January 2015

\section{KEYWORDS}

Rough set;

Multi-objective

programming;

Rough programming;

Complete-optimal

solutions;

Efficient solutions.

\begin{abstract}
This paper presents a set of multi-objective programming problems in a rough environment. These problems are classified into five classes according to the location of the roughness in the objective functions or the feasible set. We study the class in which all of the objective functions are crisp and the feasible region is a rough set and, in particular, discuss the properties of the complete and efficient (Pareto optimal) solutions of rough multi-objective programming problems. In order to obtain these solutions, we need certain theorems, which we derive. Finally, we illustrate our results by examples.
\end{abstract}

(C) 2016 Sharif University of Technology. All rights reserved.

\section{Introduction}

Multiple Objective Programming (MOP) is a research field that has been developed very much in the last three decades. It contains many real-world problems in which several objective functions have to be simultaneously optimized [1]. Since most of the realworld problems are not crisp, the methods of classical mathematics are not usually suitable for dealing with them. Almost all concepts which we use in natural language are vague or uncertain. Some of these uncertainties are expressed by interval data, fuzziness, randomness, roughness or their hybrids.

The newest theory for the joint management of vagueness and uncertainty is that of Rough Sets Theory (RST) proposed by Pawlack [2] in 1982. It is an effective theoretical framework for discussion about knowledge that has the ability to classify objects. An object in a crisp and ordinary set is completely determined, while in a rough set, it is approximately determined based on partial knowledge. In RST, any

\footnotetext{
*. Corresponding author. Tel.: +983431321313

E-mail address: Hamzehee_ali@yahoo.com (A. Hamzehee)
}

vague concept is replaced by a pair of precise concepts called the lower and the upper approximations of the vague concept. For a vague concept $X$, a lower approximation is contained in all objects which surely belong to the concept $X$ and an upper approximation contains all objects which possibly belong to the concept $X$.

In recent decades, RST has been used as the fundamental tool in many applications including optimization problems $[3,4]$. For the mathematical programming problems in the crisp environment, the aim is to maximize (minimize) an objective function over a certain set of feasible solutions. However, in many problems, the Decision-Maker (DM) may not be able to specify the objective and/or the feasible set, exactly. One of the ways by which DM can specify them is using RST. In [5], we proposed a type of linear programming problem with rough interval coefficients, and introduced two optimal ranges (surely optimal range and possibly optimal range) and two optimal solutions concepts (completely satisfactory solution and rather satisfactory solution).

Youness [6] proposed a new type of mathematical programming problem in which the decision set was a rough set and called it rough programming problem. 
He also defined two concepts for optimal solutions, namely, "surely optimal" and "possibly optimal". In his discussions, he assumed that the lower and upper approximation sets of rough sets were continuous. Osman et al. [7] classified rough programming problems into three classes according to the place of the roughness. They also defined the concepts of "rough feasibility" and "rough optimality" and studied the class of rough programming problems with a crisp objective function and a rough feasible set. This paper focuses on extending this class of problems to the case when we have multi-objective functions.

There have been numerous books, monographs, articles, and chapters in books dealing with MOP problems (see $[1,8]$ and references therein). MOP is one of the most popular methods used in Multiple-Criteria Decision-Making (MCDM). An MOP problem aims to maximize (minimize) several objective functions over a set of feasible solutions. Numerous studies of MCDM in a rough environment have been reported (for instance, see [9]). However, in this paper, we discuss MOP problems in rough environment.

In recent years, some scholars have proposed and studied different problems of operational research and decision-making, especially multi-objective programming problems, in rough environment. The newest research in this field has been developed by $\mathrm{Xu}$ and his coauthors. For example, Xu and Zhao $[10,11]$ studied a class of multi-objective decision-making problems with fuzzy rough coefficients and its application in inventory problems. $\mathrm{Xu}$ and Yao [12,13] proposed a class of multi-objective programming problems with random rough coefficients. Furthermore, Xu et al. [14] developed a Data Envelopment Analysis (DEA) model with rough parameters. Their model can be used to evaluate the performance of supply chain networks. It should be noted that in all the research, the rough coefficients or parameters of problems are "rough variables". The concept of rough variable, proposed by Liu [3], is defined via similarity relation in a rough space. While we discuss rough MOP problems using rough sets proposed by Pawlack [2] that are defined via indiscernibility relation as an equivalence relation in an approximation space. In other words, in the stated literature and most of the recent research, to solve rough programming problems, the scholars use the ranking methods such as the Expected value, $\alpha$ Optimistic value, and $\alpha$-Pessimistic value of rough variables to transform rough programming problems into deterministic programming ones. But we will state a methodology which transforms rough programming problems into classical programming problems by changing the feasible set into its lower or upper approximations.

Recently, Tao and Xu [15] proposed a new type of rough MOP problems. They investigated the proper- ties of feasible and efficient solutions and used "rough membership functions" to obtain a solution. They also obtained compromise solutions by using the interactive fuzzy satisfying method.

Li et al. [16] proposed a rough programming model based on the synthesis effect by distinguishing direct effect and indirect effect. They considered the role of equivalence classes in decision-making, which provides a useful tool for processing the roughness with different ideas. Li et al. [17] discussed the necessity and feasibility of developing a rough programming model. Their model is developed on the basis of the greatest compatible classes and synthesis effect.

In comparison with the successful application of rough set theory in real world, the research on rough programming is at the starting point. Furthermore, most of the recent achievements in rough programming have been stated based on rough variables, rough membership functions, or the effect of equivalence classes. In this paper, we further consider and analyze solutions to rough MOP problems based on upper and lower approximations of rough sets without use of rough membership functions.

The rest of the paper is organized as follows. In Section 2, some basic preliminaries about RST, MOP problems, and weighted sum method are presented. In Section 3, firstly, Single-Objective Programming (SOP) problems in rough environment are reviewed. Then, rough multi-objective programming problems are classified into five classes; and new solution concepts are defined and discussed. Finally, concluding remarks are given in Section 4.

\section{Preliminaries}

In this section, we recall some basic concepts, definitions, and theorems of RST and MOP (for more details, see $[1,2])$.

\subsection{Rough sets}

The main idea of RST is based on the indiscernibility relation that every object is associated with a certain amount of information and the object can only be expressed by means of some obtained information. Therefore, objects with the same or similar information can be indiscernible with respect to the available information. A fundamental concept in RST is the approximation space. It involves the construction of a system of definable sets.

Suppose $U$ be a finite nonempty set of objects, and $E \subseteq U \times U$ be an equivalence relation on $U$. Then, the ordered pair $A=(U, E)$ is called an approximation space generated by $E$ on $U$. The equivalence relation $E$ forms a partition on $U$ like $U / E=\left\{E_{1}, E_{2}, \ldots, E_{r}\right\}$ where for all $i=1,2, \ldots, r, E_{i} \subseteq U, E_{i} \neq \emptyset$, $E_{i} \cap E_{j}=\emptyset(i \neq j)$ and $\bigcup E_{i}=\bar{U}$. Each set $E_{i}$ 
is called an equivalence class of the approximation space $A$. In RST, every subset $M \subseteq U$ is described by the equivalence classes of $A$. Generally, two sets characterize the set $M$ called the lower and the upper approximations of $M$, which are defined by the relation $E$, respectively, as follows:

$$
\begin{aligned}
& E_{*}(M)=\bigcup\left\{E_{i} \subseteq U / E \mid E_{i} \subseteq M\right\}, \\
& E^{*}(M)=\bigcup\left\{E_{i} \subseteq U / E \mid E_{i} \cap M \neq \emptyset\right\} .
\end{aligned}
$$

Clearly, the lower approximation $E_{*}(M)$ is the greatest definable set contained in $M$, and the upper approximation $E^{*}(M)$ is the least definable set containing $M$. Also, the difference between the upper and the lower approximations is called the boundary of $M$ and is denoted by $M_{B N}=E^{*}(M)-E_{*}(M)$. If $M_{B N} \neq \emptyset$, then the set $M$ is rough (inexact), otherwise the set $M$ is crisp (exact).

\subsection{Multi-objective programming}

Any problem which optimizes multiple mathematical objective functions simultaneously under a given set of solutions is called a Multiple Objective Programming (MOP) problem and can be formulated as follows:

$$
\begin{aligned}
& \max f(x)=\left(f_{1}(x), f_{2}(x), \ldots, f_{p}(x)\right) \\
& \text { s.t. } \quad x \in X
\end{aligned}
$$

where $x=\left(x_{1}, x_{2}, \ldots, x_{n}\right)^{t}$ is an $n$-dimensional vector of decision variables, $f_{i}(x)$ for $i=1, \ldots, p$ is given realvalued objective functions, and $X$ is a feasible set of solutions.

If we directly apply the notion of optimality for SOP problem to MOP Problem (1), we obtain the following notion of a completely optimal solution.

Definition 2.1 [1]. $\quad x^{*} \in X$ is called a completely optimal solution of Problem (1) if $f_{i}\left(x^{*}\right) \geq f_{i}(x) \forall i=$ $1, \ldots, p, \forall x \in X$.

However, when the objective functions conflict with each other, a completely optimal solution does not always exist. Hence, new solution concepts, called efficient and weak efficient solutions, are introduced in MOP problems.

Definition 2.2 [1]. $\quad x^{*} \in X$ is called efficient (Pareto optimal) solution of Problem (1) if there is no other $x \in X$ such that $f_{i}(x) \geq f_{i}\left(x^{*}\right)$ for $i=1, \ldots, p$, and $f_{k}(x)>f_{k}\left(x^{*}\right)$ for at least one $k \in\{1, \ldots, p\}$.

Definition 2.3 [1]. $x^{*} \in X$ is called weak efficient solution of Problem (1) (weak Pareto optimal) if there is no $x \in X$ such that $f_{i}(x)>f_{i}\left(x^{*}\right)$ for $i=1, \ldots, p$.

\subsection{The weighted sum method}

One of the traditional approaches for solving an MOP problem to find efficient and weak efficient solutions is scalarization. A simple possible way for scalarization is the weighted sum method. This method solves the following SOP problem:

$$
\begin{aligned}
& \max \sum_{j=1}^{p} w_{j} f_{j}(x) \\
& \text { s.t. } \quad x \in X,
\end{aligned}
$$

where $w=\left(w_{1}, \ldots, w_{p}\right) \geq 0$ is a vector of weighting coefficients assigned to the objective functions by the DM.

The relationships between an optimal solution of Problem (2) with efficient and weak efficient solutions of Problem (1) are given by the following theorems.

Theorem 2.1 [1]. Let $x^{*} \in X$ be an optimal solution of Problem (2). The following statements hold.

- If $w>0$, then $x^{*}$ is an efficient solution of Problem (1);

- If $w \geq 0$, then $x^{*}$ is a weak efficient solution of Problem (1);

- If $w \geq 0$ and $x^{*}$ is a unique optimal solution of Problem (2), then $x^{*}$ is an efficient solution of Problem (1).

Theorem 2.2 [8]. In Problem (1), assume that $f_{1}, \ldots, f_{p}$ are concave functions and $X$ is a convex set. If $x^{*} \in X$ is a weak efficient solution of Problem (1), then $x^{*}$ is an optimal solution of Problem (2) for some $w \geq 0$.

\section{Single and multi-objective programming problems in rough environment}

In this section, initially, we review some definitions and theorems of rough SOP problems. Then, rough multiobjective programming problems are discussed.

\subsection{Rough single-objective programming}

A new type of programming problems in rough environment was introduced by Osman et al. [7]. They classified rough programming into three classes according to the place of roughness. In the first class, the objective function is crisp while the feasible set is a rough set [7].

Suppose that $A=(U, E)$ is an approximation space generated by an equivalence relation $E$ on the set $U$ and $U / E=\left\{E_{1}, E_{2}, \ldots, E_{r}\right\}$ is the partition generated by $E$ on $U$. Then, a rough SOP problem of the first class is as follows:

$\max f(x)$

$$
\text { s.t. } \quad x \in M
$$




$$
E_{*}(M) \subseteq M \subseteq E^{*}(M),
$$

where $M \subseteq U$ is a "rough set" in the approximation space $A=(U, E)$ as the feasible region of the problem. The sets $M^{*}=E^{*}(M)$ and $M_{*}=E_{*}(M)$ are the upper and the lower approximations of $M$ with respect to equivalence relation $E$, respectively. The function $f$ : $M^{*} \rightarrow R$ is a crisp real valued objective function which is continuous on $M^{*}$. Also, let $M_{B N}=M^{*}-M_{*}$ be boundary of $M$.

In Problem (3), the optimal value is a rough number and the optimal solutions set is a roughlydefined set (for more details about definition of rough number and rough function, see [18]).

Definition 3.1 [7]. In Problem (3), the optimal value of the objective function is a rough number denoted by $\bar{f}$, whose lower and upper bounds are denoted by $\bar{f}_{*}$ and $\bar{f}^{*}$, respectively.

To solve rough programming problems, the concepts like rough feasibility and rough optimality were defined. We quote some definitions and a theorem from [7] for Problem (3).

The solutions of Problem (3) are divided to different rough feasibility groups as follows [7]:

- A solution $x$ is surely-feasible if it belongs to the lower approximation of the feasible set, i.e. $x \in M_{*}$;

- A solution $x$ is possibly-feasible if it belongs to the upper approximation of the feasible set, i.e. $x \in M^{*}$;

- A solution $x$ is surely-infeasible if it does not belong to the upper approximation of the feasible set, i.e. $x \notin M^{*}$.

Other categories of the solutions are based on rough optimality, as follows [7]:

- A solution $x$ is surely-optimal if $f(x)=\bar{f}^{*}$;

- A solution $x$ is possibly-optimal if $f(x) \geq \bar{f}_{*}$;

- A solution $x$ is surely-not optimal if $f(x)<\bar{f}_{*}$.

In order to compute the lower and upper bounds for the optimal value of objective function $\bar{f}$, we recall the following theorem from [7].

Theorem 3.1 [7]. In Problem (3), the lower and upper bounds of the optimal objective value $\bar{f}$ are given by:

$$
\bar{f}_{*}=\sup \{a, b\} \text { and } \bar{f}^{*}=\sup \{a, c\},
$$

where (assuming the existence of the solution to the following crisp problems):

$$
\begin{aligned}
& a=\max _{x \in M_{*}} f(x), \\
& c=\max _{x \in M_{B N}} f(x),
\end{aligned}
$$

$$
b=\sup _{\substack{E_{i} \in U / E \\ E_{i} \subseteq M_{B N}}}\left\{\min _{x \in E_{i}} f(x)\right\} .
$$

Definition 3.2 [7]. In Problem (3), there are four optimal sets covering all possible degrees of feasibility and optimality, as follows:

- $F O_{s s}=\left\{x \in M_{*} \mid f(x)=\bar{f}^{*}\right\}$ is the set of all surely-feasible and surely-optimal solutions;

- $F O_{s p}=\left\{x \in M_{*} \mid f(x) \geq \bar{f}_{*}\right\}$ is the set of all surely-feasible and possibly-optimal solutions;

- $F O_{p s}=\left\{x \in M^{*} \mid f(x)=\bar{f}^{*}\right\}$ is the set of all possibly-feasible and surely-optimal solutions;

- $F O_{p p}=\left\{x \in M^{*} \mid f(x) \geq \bar{f}_{*}\right\}$ is the set of all possibly-feasible and possibly-optimal solutions.

\subsection{Rough multi-objective programming}

In this section, the MOP problems in rough environment are investigated. We call them Rough Multiple Objective Programming (RMOP) problems, where the roughness may appear in different ways. In other words, the feasible set and/or the objective functions may be rough or crisp. Thus, RMOP problems can be classified as follows:

(a) MOP problems with rough feasible set and crisp objective functions;

(b) MOP problems with crisp feasible set and rough objective functions;

(c) MOP problems with rough feasible set and rough objective functions;

(d) MOP problems with crisp feasible set and both crisp and rough objective functions;

(e) MOP problems with rough feasible set and both crisp and rough objective functions.

To solve RMOP problems, we use the concepts of rough feasibility and rough optimality based on the results of Osman et al. [7] for rough SOP problems. However, we only consider an ROMP problem of the first class (a), where the objective functions are crisp and feasible set is rough.

Similar to rough SOP problems, let $A=(U, E)$ and $U / E=\left\{E_{1}, E_{2}, \ldots, E_{r}\right\}$ be the approximation space and the partition generated by equivalence relation $E$ on the set $U$, respectively. Then, the problem can be formulated as follows:

$$
\begin{gathered}
\max \quad f(x)=\left(f_{1}(x), f_{2}(x), \ldots, f_{p}(x)\right) \\
\text { s.t. } \quad x \in M \\
E_{*}(M) \subseteq M \subseteq E^{*}(M),
\end{gathered}
$$

where $M \subseteq U$ is a Rough set as the feasible region of Problem (4). The sets $M_{*}=E_{*}(M), M^{*}=E^{*}(M)$, 
and $M_{B N}$ have the same definitions as those in Problem (3) of Section 3.1. For all $j=1,2, \ldots, p$, the functions $f_{j}: M^{*} \rightarrow R$ are crisp real valued objective functions, which are continuous on $M^{*}$.

Firstly, the following example (taken from [6] with small modifications) is presented as a real problem to show an instance in Problem (4).

Example 3.1. Assume the drugs $D 1$ and $D 2$ were developed to treat liver cancer. Let $f$ and $g$ be the effects of the drugs $D 1$ and $D 2$, respectively. In other words, if $U$ is the set of all cells of the liver then $f$ and $g$ are two functions of $U$ on the real numbers set $R$. Moreover, the values $f(x)$ and $g(x)$ mean the effects of $D 1$ and $D 2$ on the cell $x$ of $U$, respectively. Suppose:

1. Using drugs for a year implies to kill $30 \%$ of cancer cells;

2. Using drugs for 2 years implies to kill $50 \%$ of cancer cells and $1 \%$ of normal cells;

3. Using drugs for 3 years implies to kill $70 \%$ of cancer cells and $2 \%$ of normal cells;

4. Using drugs for 4 years implies to kill $99 \%$ of cancer cells and $3 \%$ of normal cells.

Equivalence relation $E$ on $U$ is defined as follows:

$$
(u, v) \in E \Leftrightarrow\left\{\begin{array}{l}
u \text { and } v \text { are killed in the same year } \\
\text { or } \\
u \text { and } v \text { are not killed in the end of } \\
\quad \text { treatment. }
\end{array}\right.
$$

Now, let $M \subset U$ be the set of all cancer cells. The defined equivalence relation $E$ makes a partition to $U$ as:

$$
\begin{aligned}
E_{1}= & \{u \in M \mid u \text { was killed in the first year }\}, \\
E_{2}= & \{u \in M, v \in U \backslash M \mid u \text { and } v \text { were killed in } \\
& \text { the second year }\}, \\
E_{3}= & \{u \in M, v \in U \backslash M \mid u \text { and } v \text { were killed in } \\
& \text { the third year }\}
\end{aligned}
$$$$
E_{4}=\{u \in M, v \in U \backslash M \mid u \text { and } v \text { were killed in }
$$$$
\text { the fourth year\}, }
$$$$
E_{5}=\{v \in U \backslash M \mid v \text { were not killed in }
$$

the end of treatment\}.

Then, set $M$ is a rough set with respect to equivalence relation $E$, such that the lower and upper approximations of $M$ are $E_{*}(M)=E_{1}$ and $E^{*}(M)=E_{1} \cup E_{2} \cup$ $E_{3} \cup E_{4}$, respectively.

The problem of finding the maximum effect of each drug $D 1$ and $D 2$, is a rough bi-objective programming and is formulated as follows:

$$
\begin{array}{ll}
\max & f_{t}(x) \\
\max & g_{t}(x) \\
\text { s.t. } & x \in M \\
& E_{*}(M) \subseteq M \subseteq E^{*}(M),
\end{array}
$$

where $f_{t}, g_{t}: M \rightarrow R$ are the effects of the drugs on cell $x \in M$ in a certain year $t \in\{1,2,3,4\}$.

With regard to the rough $\mathrm{SOP}$, we present the next definition.

Definition 3.3. In Problem (4), the optimal value of the objective function is a rough number denoted by $\bar{f}=\left(\bar{f}_{1}, \bar{f}_{2}, \ldots, \bar{f}_{p}\right)$, whose lower and upper bounds are denoted by $\bar{f}_{*}=\left(\bar{f}_{1 *}, \bar{f}_{2 *}, \ldots, \bar{f}_{p_{*}}\right)$ and $\bar{f}^{*}=$ $\left(\bar{f}_{1}^{*}, \bar{f}_{2}^{*}, \ldots, \bar{f}_{p}^{*}\right)$, respectively.

Remark 3.1. If $\bar{f}_{*}=\bar{f}^{*}$, i.e. $\bar{f}_{j_{*}}=\bar{f}_{j}^{*}$, for all $j=$ $1,2, \ldots, p$, the optimal value is exact, otherwise it is rough.

In order to deal with Problem (4), we need to find the optimal values and solutions for each rough SOP Problem (5), for all $j=1,2, \ldots, p$ :

$$
\begin{array}{ll}
\max & f_{j}(x) \\
\text { s.t. } & x \in M \\
& E_{*}(M) \subseteq M \subseteq E^{*}(M) .
\end{array}
$$

With regard to Section 3.1, the lower and upper bounds of each optimal objective value $\bar{f}_{j}$ for Problem (5) are given by:

$$
\bar{f}_{j_{*}}=\sup \left\{a_{j}, b_{j}\right\} \text { and } \bar{f}_{j}^{*}=\sup \left\{a_{j}, c_{j}\right\},
$$

where (assuming the existence of the solution to the following crisp problems):

$$
\begin{aligned}
& a_{j}=\max _{x \in M_{*}} f_{j}(x), \\
& c_{j}=\max _{x \in M_{B N}} f_{j}(x) \text { and } \\
& b_{j}=\sup _{\substack{E_{i} \in U / E \\
E_{i} \subseteq M_{B N}}}\left\{\min _{x \in E_{i}} f_{j}(x)\right\} .
\end{aligned}
$$

Moreover, we denote the set of all "surely-feasible 
and surely-optimal", "surely-feasible and possiblyoptimal", "possibly-feasible and surely-optimal", and "possibly-feasible and possibly-optimal" solutions of Problem (5) by $F O_{s s}^{j}, F O_{s p}^{j}, F O_{p s}^{j}$, and $F O_{p p}^{j}$, respectively.

In what follows, we extend the notions of feasibility and optimality of rough SOP problems to the multiple case.

Definition 3.4. For Problem (4):

- $\hat{x}$ is a surely-complete optimal solution if $f_{j}(\hat{x})=$ $\bar{f}_{j}^{*}$, for all $j=1, \ldots, p$;

- $\hat{x}$ is a possibly-complete optimal solution if $f_{j}(\hat{x}) \geq$ $\bar{f}_{j_{*}}$, for all $j=1, \ldots, p$.

Similar to Definition 3.2 , we can define four optimal sets covering all possible degrees of feasibility and complete optimality as follows:

- $F O_{s(s c)}=\left\{x \in M_{*} \mid f_{j}(x)=\bar{f}_{j}^{*}, j=1, \ldots, p\right\}$ is the set of all surely-feasible and surely-complete optimal solutions;

- $F O_{s(p c)}=\left\{x \in M_{*} \mid f_{j}(x) \geq \bar{f}_{j_{*}}, j=1, \ldots, p\right\}$ is the set of all surely-feasible and possibly-complete optimal solutions;

- $F O_{p(s c)}=\left\{x \in M^{*} \mid f_{j}(x)=\bar{f}_{j}^{*}, j=1, \ldots, p\right\}$ is the set of all possibly-feasible and surely-complete optimal solutions;

- $F O_{p(p c)}=\left\{x \in M^{*} \mid f_{j}(x) \geq \bar{f}_{j_{*}}, j=1, \ldots, p\right\}$ is the set of all possibly-feasible and possibly-complete optimal solutions.

Proposition 3.1. For Problem (4), the following statements hold:

(i) $F O_{s(s c)}=\bigcap_{j=1}^{p} F O_{s s}^{j}$;

(ii) $F O_{s(p c)}=\bigcap_{j=1}^{p} F O_{s p}^{j}$;

(iii) $F O_{p(s c)}=\bigcap_{j=1}^{p} F O_{p s}^{j}$;

(iv) $F O_{p(p c)}=\bigcap_{j=1}^{p} F O_{p p}^{j}$.

Proof. The proof is straight forward.

Often, the sets $F O_{s(s c)}, F O_{s(p c)}$ and $F O_{p(s c)}$ are empty. In other words, surely- and possibly-complete optimal solutions do not always exist. Therefore, we introduce another concepts instead of complete optimal solutions for RMOP problems. These solution concepts are based on efficiency (Pareto optimality). To find surely- and possibly-efficient solutions, we use the union of the sets $F O_{s s}^{j}, F O_{s p}^{j}, F O_{p s}^{j}$, and $F O_{p p}^{j}$. Hence, for Problem (4), the following notations are used:

$$
P=\bigcup_{j=1}^{p} F O_{s s}^{j}, \quad Q=\bigcup_{j=1}^{p} F O_{s p}^{j},
$$

$$
S=\bigcup_{j=1}^{p} F O_{p s}^{j}, \quad \text { and } \quad T=\bigcup_{j=1}^{p} F O_{p p}^{j} .
$$

Definition 3.5. For Problem (4):

- $\hat{x} \in M$ is called a surely-efficient solution if: $\hat{x} \in M_{*}$ and there is no other $x \in M_{*}$ such that $f_{i}(x) \geq f_{i}(\hat{x})$ for $i=1, \ldots, p$, and $f_{k}(x)>f_{k}(\hat{x})$ for at least one $k \in\{1, \ldots, p\}$;

- $\hat{x} \in M$ is called a possibly-efficient solution if: $\hat{x} \in M^{*}$ and there is no other $x \in M^{*}$ such that $f_{i}(x) \geq f_{i}(\hat{x})$ for $i=1, \ldots, p$, and $f_{k}(x)>f_{k}(\hat{x})$ for at least one $k \in\{1, \ldots, p\}$.

We show that if the sets $P, Q, S$, and $T$ are nonempty, then the surely and possibly-efficient solutions belong to these sets. Therefore, the sets $M_{*}$ and $M^{*}$ in Definition 3.5 can be restricted to $P($ or $Q)$ and $S($ or $T$ ), respectively.

Proposition 3.2. For Problem (4), the following statements hold:

- If $P \neq \emptyset$, then each member of $M_{*} \backslash P$ cannot be a surely-efficient solution;

- If $Q \neq \emptyset$, then each member of $M_{*} \backslash Q$ cannot be a possibly-efficient solution;

- If $S \neq \emptyset$, then each member of $M^{*} \backslash S$ cannot be a surely-efficient solution;

- If $T \neq \emptyset$, then each member of $M^{*} \backslash T$ cannot be a possibly-efficient solution.

Proof. We prove only the first item. The other items can be proved similarly. It should be proved that for every $x \in M_{*} \backslash P$, there exists $\hat{x} \in P$ such that $f_{i}(\hat{x}) \geq$ $f_{i}(x)$ for $i=1, \ldots, p$, and $f_{k}(\hat{x})>f_{k}(x)$ for at least one $k \in\{1, \ldots, p\}$, or equivalently:

$$
\begin{aligned}
& \left(f_{i}(\hat{x}) \geq f_{i}(x) \text { for } i=1, \ldots, p\right) \text { and } \\
& \left(f_{1}(\hat{x}), \ldots, f_{p}(\hat{x})\right) \neq\left(f_{1}(x), \ldots, f_{p}(x)\right)
\end{aligned}
$$

To this end, on the contrary suppose that there is $\bar{x} \in$ $M_{*} \backslash P$ such that for all $x \in P$ :

$$
\begin{aligned}
& \left(\exists j \in\{1, \ldots, p\}: f_{j}(x)<f_{j}(\bar{x})\right) \text { or } \\
& \left(f_{1}(x), \ldots, f_{p}(x)\right)=\left(f_{1}(\bar{x}), \ldots, f_{p}(\bar{x})\right) .
\end{aligned}
$$

Firstly, if $f_{j}(x)<f_{j}(\bar{x})$ for $x \in P$, then $f_{j}(x)=\bar{f}_{j}^{*}=$ $\sup \left\{a_{j}, c_{j}\right\}<f_{j}(\bar{x})$. The following two cases can be considered:

(i) If $a_{j} \geq c_{j}$, then $\bar{f}_{j}^{*}=a_{j}=\max _{x \in M_{*}} f_{j}(x)<f_{j}(\bar{x})$, which is a contradiction since $\bar{x} \in M_{*} \backslash P \subseteq M_{*}$. 
(ii) If $a_{j}<c_{j}$, then $\max _{x \in M_{*}} f_{j}(x)=a_{j}<c_{j}=\bar{f}_{j}^{*}<$ $f_{j}(\bar{x})$, which is a contradiction since $\bar{x} \in M_{*}$.

Secondly, if $\left(f_{1}(x), \ldots, f_{p}(x)\right)=\left(f_{1}(\bar{x}), \ldots, f_{p}(\bar{x})\right)$ then $f_{j}(\bar{x})=\bar{f}_{j}^{*}$ for $j=1, \ldots, p$, since $x \in P$ and $f_{j}(x)=\bar{f}_{j}^{*}$ for $j=1, \ldots, p$. Thus, $\bar{x} \in F O_{s s}^{j}$ for $j=$ $1, \ldots, p$. So, $\bar{x} \in \bigcap_{j=1}^{p} F O_{s s}^{j} \subseteq \bigcup_{j=1}^{p} F O_{s s}^{j}=P$, which is a contradiction since $\bar{x} \in M_{*} \backslash P$. This completes the proof. $\square$

According to Proposition 3.2, if the sets $P, Q, S$, and $T$ are nonempty, then the rough efficient solutions of RMOP problems are restricted to these sets. Hence, we can modify Definition 3.5 as Definition 3.6 to cover all possible degrees of feasibility. It should be noted that usually among the sets $P, Q, S$ and $T$, only the set $P$ is empty.

Definition 3.6. For Problem (4):

- $\bar{x}$ is called a surely-feasible and surely-efficient solution if:

$\bar{x} \in P$ and there is no other $x \in P$ such that $f_{i}(x) \geq f_{i}(\bar{x})$ for $i=1, \ldots, p$, and $f_{k}(x)>f_{k}(\bar{x})$ for at least one $k \in\{1, \ldots, p\}$;

- $\bar{x}$ is called a surely-feasible and possibly-efficient solution if:

$\bar{x} \in Q$ and there is no other $x \in Q$ such that $f_{i}(x) \geq f_{i}(\bar{x})$ for $i=1, \ldots, p$, and $f_{k}(x)>f_{k}(\bar{x})$ for at least one $k \in\{1, \ldots, p\}$;

- $\bar{x}$ is called a possibly-feasible and surely-efficient solution if:

$\bar{x} \in S$ and there is no other $x \in S$ such that $f_{i}(x) \geq f_{i}(\bar{x})$ for $i=1, \ldots, p$, and $f_{k}(x)>f_{k}(\bar{x})$ for at least one $k \in\{1, \ldots, p\}$;

- $\bar{x}$ is called a possibly-feasible and possibly-efficient solution if:

$\bar{x} \in T$ and there is no other $x \in T$ such that $f_{i}(x) \geq$ $f_{i}(\bar{x})$ for $i=1, \ldots, p$, and $f_{k}(x)>f_{k}(\bar{x})$ for at least one $k \in\{1, \ldots, p\}$.

Remark 3.2. If the relations $\geq$ of all items in the Definition 3.6 are replaced by $>$, then the expression "efficient" should be replaced with "weak efficient". For instance:

- $\bar{x}$ is called a surely-feasible and possibly-weak efficient solution of (4) if:

$\bar{x} \in Q$ and there is no other $x \in Q$ such that $f_{i}(x)>f_{i}(\bar{x})$ for $i=1, \ldots, p$.

Notation. We denote the efficient sets in terms of four optimal sets covering all possible degrees of feasibility and optimality of the solutions as:

- $F O_{s(s e)}$ : The set of all surely-feasible and surelyefficient solutions;
- $F O_{s(p e)}$ : The set of all surely-feasible and possiblyefficient solutions;

- $F O_{p(s e)}$ : The set of all possibly-feasible and surelyefficient solutions;

- $F O_{p(p e)}$ : The set of all possibly-feasible and possibly-efficient solutions.

Proposition 3.3. For Problem (4), the following statements hold:

(i) $F O_{s(s c)} \subseteq F O_{s(s e)}$;

(ii) $F O_{s(p c)} \subseteq F O_{s(p e)}$;

(iii) $F O_{p(s c)} \subseteq F O_{p(s e)}$;

(iv) $F O_{p(p c)} \subseteq F O_{p(p e)}$.

Proof. The proof is straight forward. $\square$

As mentioned before, the traditional approach to solve an MOP problem is scalarization and one of the most popular scalarization methods is the weighted sum method. The following theorems are stated for finding surely and possibly-efficient solutions of the RMOP problems.

Theorem 3.2. Let $x^{*}$ be an optimal solution of the crisp Problem (6). If $w_{j}>0$ for $j=1, \ldots, p$, then $x^{*}$ is a surely-feasible and surely-efficient solution of Problem (4).

$$
\begin{array}{ll}
\max & \sum_{j=1}^{p} w_{j} f_{j}(x) \\
\text { s.t. } & x \in M_{*} \\
& f_{r}(x)=\bar{f}_{r}{ }^{*},
\end{array}
$$

where $r \in\{1, \ldots, p\}$.

Proof. Since $x^{*}$ is an optimal solution of Problem (6), then it is feasible. Thus, $x^{*} \in M_{*}$ and $f_{r}\left(x^{*}\right)=\bar{f}_{r}{ }^{*}$. So, $x^{*} \in P=\bigcup_{j=1}^{p} F O_{s s}^{j}$. Now, on the contrary suppose that there is $\bar{x} \in P$ such that $f_{i}(\bar{x}) \geq f_{i}\left(x^{*}\right)$ for $i=1, \ldots, p$, and $f_{k}(\bar{x})>f_{k}\left(x^{*}\right)$ for at least one $k \in\{1, \ldots, p\}$. If $r=k$, then $f_{r}(\bar{x})>f_{r}\left(x^{*}\right)=\bar{f}_{r}{ }^{*}$. Therefore, $f_{r}(\bar{x})>\bar{f}_{r}{ }^{*}=\sup \left\{a_{r}, c_{r}\right\}$ and $\bar{x} \in P \subseteq M_{*}$, which is a contradiction. If $r \neq k$, then $f_{r}(\bar{x}) \geq$ $f_{r}\left(x^{*}\right)=\bar{f}_{r}{ }^{*}$. Since $\bar{x} \in P$, then $\bar{x} \in M_{*}$ and $f_{r}(\bar{x})=$ $\bar{f}_{r}{ }^{*}$. Therefore, $\bar{x}$ is a feasible solution to Problem (6). On the other hand, $w_{i} f_{i}(\bar{x}) \geq w_{i} f_{i}\left(x^{*}\right)$ for $i=1, \ldots, p$ and $w_{k} f_{k}(\bar{x})>w_{k} f_{k}\left(x^{*}\right)$. Thus, $\sum_{j=1}^{p} w_{j} f_{j}(\bar{x})>$ $\sum_{j=1}^{p} w_{j} f_{j}\left(x^{*}\right)$ which is a contradiction.

Theorem 3.3. Let $x^{*}$ be an optimal solution of the crisp Problem (7). If $w_{j}>0$ for $j=1, \ldots, p$, then 
$x^{*}$ is a surely-feasible and possibly-efficient solution to Problem (4).

$$
\begin{array}{ll}
\max & \sum_{j=1}^{p} w_{j} f_{j}(x) \\
\text { s.t. } & x \in M_{*} \\
& f_{r}(x) \geq \bar{f}_{r *},
\end{array}
$$

where $r \in\{1, \ldots, p\}$.

Proof. Since $x^{*}$ is an optimal solution to Problem (7), it is feasible. Thus, $x^{*} \in M_{*}$ and $f_{r}\left(x^{*}\right) \geq \bar{f}_{r_{*}}$. So, $x^{*} \in Q=\bigcup_{j=1}^{p} F O_{s p}^{j}$. Now, on the contrary suppose that there is $\bar{x} \in Q$ such that $f_{i}(\bar{x}) \geq f_{i}\left(x^{*}\right)$ for $i=1, \ldots, p$, and $f_{k}(\bar{x})>f_{k}\left(x^{*}\right)$ for at least one $k \in\{1, \ldots, p\}$. Firstly, since $x^{*}$ is feasible for Problem (7), if $r \neq k$, then $f_{r}(\bar{x}) \geq f_{r}\left(x^{*}\right) \geq \bar{f}_{r_{*}}$. If $r=k$, then $f_{r}(\bar{x})>f_{r}\left(x^{*}\right) \geq \bar{f}_{r_{*}}$ and $\bar{x} \in Q \subseteq M_{*}$. Thus, $\bar{x}$ is a feasible solution to Problem (7). Secondly, $w_{i} f_{i}(\bar{x}) \geq$ $w_{i} f_{i}\left(x^{*}\right)$ for $i=1, \ldots, p$ and $w_{k} f_{k}(\bar{x})>w_{k} f_{k}\left(x^{*}\right)$. Therefore, $\sum_{j=1}^{p} w_{j} f_{j}(\bar{x})>\sum_{j=1}^{p} w_{j} f_{j}\left(x^{*}\right)$. This is a contradiction to the fact that $x^{*}$ is the optimal solution to Problem (7).

Theorem 3.4. Let $x^{*}$ be an optimal solution of the crisp Problem (8). If $w_{j}>0$ for $j=1, \ldots, p$, then $x^{*}$ is a possibly-feasible and surely-efficient solution to Problem (4).

$$
\begin{array}{ll}
\max & \sum_{j=1}^{p} w_{j} f_{j}(x) \\
\text { s.t. } \quad & x \in M^{*} \\
& f_{r}(x)=\bar{f}_{r}^{*},
\end{array}
$$

where $r \in\{1, \ldots, p\}$.

Proof. The proof is similar to that of Theorem 3.2.

Theorem 3.5. Let $x^{*}$ be an optimal solution of the crisp Problem (9). If $w_{j}>0$ for $j=1, \ldots, p$, then $x^{*}$ is a possibly-feasible and possibly-efficient solution to Problem (4).

$$
\begin{array}{ll}
\max & \sum_{j=1}^{p} w_{j} f_{j}(x) \\
\text { s.t. } & x \in M^{*} \\
& f_{r}(x) \geq \bar{f}_{r_{*}},
\end{array}
$$

where $r \in\{1, \ldots, p\}$.
Proof. The proof is similar to that of Theorem 3.3. $\square$

It should be noted that in Theorems 3.2-3.5 if the solution $x^{*}$ is a unique optimal solution to the weighted crisp Problems (6)-(9), then $w_{j}$ can be nonnegative $\left(w_{j} \geq 0\right)$ for $j=1, \ldots, p$.

Moreover, if the uniqueness is not guaranteed and $w_{j} \geq 0$ for $j=1, \ldots, p$, then the optimal solution of the weighted crisp Problems (6)-(9) is a "weak efficient" solution to Problem (4) with different degrees of feasibility. For instance:

Theorem 3.6. Let $x^{*}$ be an optimal solution to the crisp Problem (7). If $w_{j} \geq 0$ for $j=1, \ldots, p$, then $x^{*}$ is a surely-feasible and possibly weak-efficient solution to Problem (4).

Proof. The proof is similar to that of Theorem 3.3. $\square$ Also, in order to find surely- and possibly complete optimal solutions of the RMOP Problem (4), we can solve some crisp problems similar to Problems (6)(9).

Theorem 3.7. Let $x^{*}$ be an optimal solution to the crisp Problem (10). If $w_{j}>0$ for $j=1, \ldots, p$, then $x^{*} \in F O_{s(p c)}$, i.e. $x^{*}$ is a surely-feasible and possiblycomplete optimal solution to Problem (4).

$$
\begin{aligned}
\max & \sum_{j=1}^{p} w_{j} f_{j}(x) \\
\text { s.t. } & \quad x \in M_{*} \\
& f_{r}(x) \geq \bar{f}_{r_{*}} \quad r=1,2, \ldots, p .
\end{aligned}
$$

Proof. Since $x^{*}$ is an optimal solution to Problem (10), it is feasible. Thus, $x^{*} \in M_{*}$ and $f_{r}\left(x^{*}\right) \geq$ $\bar{f}_{r_{*}} \forall r=1,2, \ldots, p$. Subsequently, $x^{*} \in \bigcap_{j=1}^{p} F O_{s p}^{j}=$ $F O_{s(p c)}$

Finally, we study the converse of Theorems 3.23.6. As an instance, we state only the converse of Theorem 3.6. To do so, we need the following theorem which is taken from [1].

Theorem 3.8 [1]. Let $M$ be a convex set and $f_{j}$ : $M \rightarrow R$ be concave function for $j=1, \ldots, p$. Then, if the system $f_{j}>0, j=1, \ldots, p$ has no solution $x \in M$, there exist $w=\left(w_{1}, \ldots, w_{p}\right) \geq 0, \quad \sum_{j=1}^{p} w_{j}=1$ such that $\forall x \in M, \sum_{j=1}^{p} w_{j} f_{j}(x) \leq 0$.

Theorem 3.9. Suppose $M_{*}$ be a convex set and $f_{j}$ : $M^{*} \rightarrow R$ be concave function for $j=1,2, \ldots, p$. If $Q \neq \emptyset$ and $\hat{x}$ is a surely-feasible and possibly-weak efficient solution to Problem (4), then there exist $w=$ $\left(w_{1}, \ldots, w_{p}\right) \geq 0$ such that $\hat{x}$ is an optimal solution to the crisp Problem (7). 
Proof. Since $\hat{x}$ is a surely-feasible and possibly-weak efficient solution to Problem (4), then $\hat{x} \in Q \subseteq M_{*}$ and subsequently there exists $r \in\{1, \ldots, p\}$ such that $f_{r}(\hat{x}) \geq \bar{f}_{r_{*}}$. Thus, $\hat{x}$ is a feasible solution for Problem (7). Moreover, there is no other $x \in Q$ such that $f_{j}(x)>f_{j}(\hat{x})$ for $j=1, \ldots, p$. This means that the system $f_{j}(x)-f_{j}(\hat{x})>0, j=1,2, \ldots, p$ has no solution $x \in Q \subseteq M_{*}$. Therefore, by Theorem 3.7, there exist $w=\left(w_{1}, \ldots, w_{p}\right) \geq 0, \sum_{j=1}^{p} w_{j}=1$ such that for all $x \in M_{*}$, we have $\sum_{j=1}^{p} w_{j}\left(f_{j}(x)-f_{j}(\hat{x})\right) \leq 0$. Thus, $\sum_{j=1}^{p} w_{j} f_{j}(x) \leq \sum_{j=1}^{p} w_{j} f_{j}(\hat{x})$ for all $x \in M_{*}$. Hence, $\hat{x}$ is an optimal solution to Problem (7).

In the sequel, some numerical examples are given to illustrate some of the theoretical results.

Example 3.2. Let $U=\left\{x=\left(x_{1}, x_{2}\right) \in R^{2} \mid\left(x_{1}-\right.\right.$ $\left.3)^{2}+\left(x_{2}-3\right)^{2} \leq 9\right\}$, and let $K$ be a polytope generated by the following closed half-spaces:

$$
\begin{aligned}
& g_{1}(x)=x_{1}+x_{2}-8 \leq 0, \\
& g_{2}(x)=x_{1}-x_{2}-2 \leq 0, \\
& g_{3}(x)=x_{1}+x_{2}-4 \geq 0, \\
& g_{4}(x)=x_{1}-x_{2}+2 \geq 0 .
\end{aligned}
$$

Suppose that $E$ is an equivalence relation on $U$ such that $U / E=\left\{E_{1}, E_{2}, E_{3}\right\}$ where:

$$
\begin{aligned}
& E_{1}=\{x \in U: x \text { is an interior point of polytope } K\}, \\
& E_{2}=\{x \in U: x \text { is a boundary point of polytope } K\}, \\
& E_{3}=\{x \in U: x \text { is an exterior point of polytope } K\} .
\end{aligned}
$$

Now, assume that $M$ is a rough feasible region in the approximation space $A=(U, E)$ such that $M_{*}=$ $E_{1} \cup E_{2}$ and $M^{*}=E_{1} \cup E_{2} \cup E_{3}$ are lower and upper approximations of $M$, respectively. Also, the boundary of $M$ is given by $M_{B N}=E_{3}$ (see Figure 1). The structure of $M$ is taken from $[7,6]$. Consider the following RMOP problem:

$$
\begin{array}{ll}
\max & f_{1}(x)=\left(x_{1}-4\right)^{2}+\left(x_{2}-2\right)^{2}-2 \\
\max & f_{2}(x)=x_{1}^{2}+x_{2}^{2}-5 x_{1} x_{2} \\
\max & f_{3}(x)=5-\left(x_{1}-1\right)^{2}-\left(x_{2}-3\right)^{2} \\
\text { s.t. } & x \in M \\
& M_{*} \subseteq M \subseteq M^{*} .
\end{array}
$$

In order to find some efficient solutions to Problem (11), we solve some crisp SOP problems by Theorems 3.1-3.5.

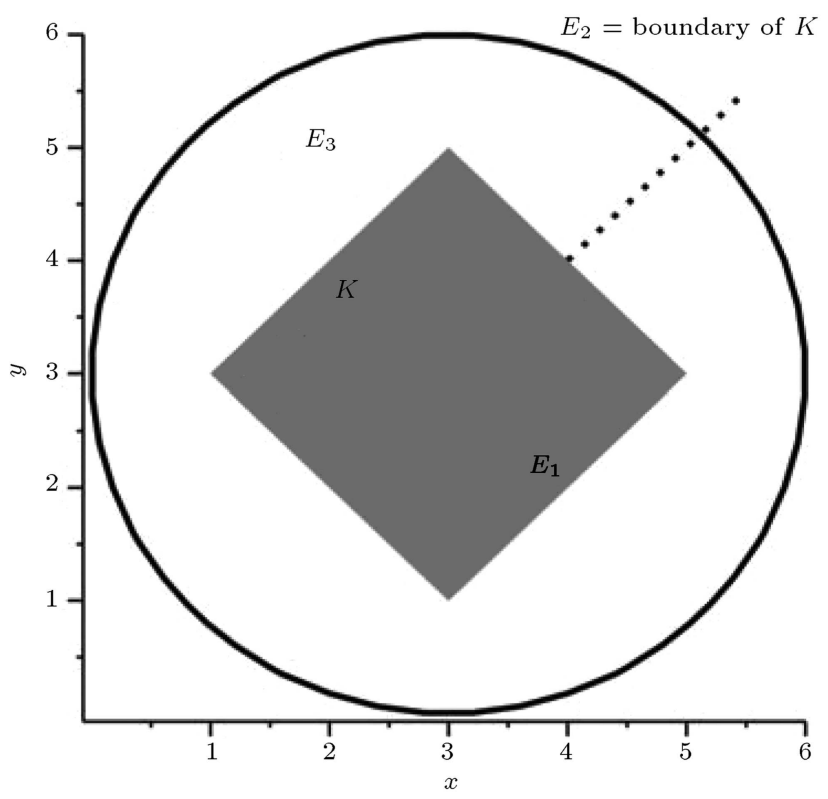

Figure 1. The set $K$ and equivalence classes of $E$ on $U$.

First step: Finding the rough optimal value $\bar{f}=$ $\left(\bar{f}_{1}, \bar{f}_{2}, \bar{f}_{3}\right)$ :

$$
\begin{aligned}
\bar{f}_{1 *} & =a_{1}=f_{1}(1,3)=f_{1}(3,5)=8, \\
\bar{f}_{1}^{*} & =c_{1}=f_{1}(0.8787,5.1213)=17.4853, \\
\bar{f}_{2_{*}} & =a_{2}=f_{2}(1,3)=f_{2}(3,1)=-5, \\
\bar{f}_{2}^{*} & =c_{2}=f_{2}(4.0209,0.1791)=12.6, \\
\bar{f}_{3 *} & =\bar{f}_{3}^{*}=a_{3}=f_{3}(1,3)=5\left(c_{3}=f_{3}(1-\epsilon, 3-\epsilon)\right. \\
& \left.=5-\epsilon<a_{3}\right) .
\end{aligned}
$$

Second step: Finding the rough optimal sets for any objective function:

$$
\begin{aligned}
F O_{s s}^{1} & =\left\{x \in M_{*} \mid f_{1}(x)=\bar{f}_{1}^{*}=17.4853\right\}=\emptyset, \\
F O_{s p}^{1} & =\left\{x \in M_{*} \mid f_{1}(x) \geq \bar{f}_{1 *}=8\right\}=\{(1,3),(3,5)\}, \\
F O_{p s}^{1} & =\left\{x \in M^{*} \mid f_{1}(x)=\bar{f}_{1}^{*}=17.4853\right\} \\
& =\{(0.8787,5.1213)\}, \\
F O_{p p}^{1} & =\left\{x \in M^{*} \mid f_{1}(x) \geq \bar{f}_{1 *}=8\right\} \\
& =\left\{\left(x_{1}, x_{2}\right) \in U:\left(x_{1}-4\right)^{2}+\left(x_{2}-2\right)^{2} \geq 10\right\}, \\
F O_{s s}^{2} & =\left\{x \in M_{*} \mid f_{2}(x)=\bar{f}_{2}^{*}=12.6\right\}=\emptyset, \\
F O_{s p}^{2} & =\left\{x \in M_{*} \mid f_{2}(x) \geq \bar{f}_{2 *}=-5\right\}=\{(1,3),(3,1)\},
\end{aligned}
$$




$$
\begin{aligned}
F O_{p s}^{2} & =\left\{x \in M^{*} \mid f_{2}(x)=\bar{f}_{2}^{*}=12.6\right\} \\
& =\{(4.0209,0.1791)\} \\
F O_{p p}^{2} & =\left\{x \in M^{*} \mid f_{2}(x) \geq \bar{f}_{2 *}=-5\right\} \\
& =\left\{\left(x_{1}, x_{2}\right) \in U: x_{1}^{2}+x_{2}^{2}-5 x_{1} x_{2} \geq-5\right\} .
\end{aligned}
$$

Finally, since $c_{3}<a_{3}, \quad F O_{s s}^{3}=F O_{s p}^{3}=F O_{p s}^{3}=$ $F O_{p p}^{3}=\{(1,3)\}$.

Third step: Finding the rough completely optimal sets:

$$
\begin{aligned}
& F O_{s(s c)}=\bigcap_{j=1}^{3} F O_{s s}^{j}=\emptyset, \\
& F O_{s(p c)}=\bigcap_{j=1}^{3} F O_{s p}^{j}=\{(1,3)\}, \\
& F O_{p(s c)}=\bigcap_{j=1}^{3} F O_{p s}^{j}=\emptyset, \\
& F O_{p(p c)}=\bigcap_{j=1}^{3} F O_{p p}^{j}=\{(1,3)\} .
\end{aligned}
$$

Therefore, Problem (11) has no surely-complete optimal solution. But, the point $x^{*}=(1,3)$ is a possiblycomplete optimal solution.

Fourth step: Finding the rough efficient solutions sets, firstly, the sets $P, Q, S$, and $T$ are as follows:

$$
\begin{aligned}
& P=\bigcup_{j=1}^{3} F O_{s s}^{j}=\{(1,3)\}, \\
& Q= \bigcup_{j=1}^{3} F O_{s p}^{j}=\{(1,3),(3,5),(3,1)\}, \\
& S=\bigcup_{j=1}^{3} F O_{p s}^{j}=\{(0.8787,5.1213),(4.0209,0.1791),(1,3)\}, \\
& T=\bigcup_{j=1}^{3} F O_{p p}^{j}=\left\{x \in U:\left\{\left(x_{1}-4\right)^{2}+\left(x_{2}-2\right)^{2} \geq 10\right\}\right. \\
&\left.\cup\left\{x_{1}^{2}+x_{2}^{2}-5 x_{1} x_{2} \geq-5\right\}\right\} .
\end{aligned}
$$

Secondly, according to Theorems 3.2-3.5, we solve the SOP problems $P 1, P 2, P 3$, and $P 4$ as follows:

$P 1$ :

$$
\begin{array}{cl}
\max & w_{1}\left(\left(x_{1}-4\right)^{2}+\left(x_{2}-2\right)^{2}-2\right) \\
& +w_{2}\left(x_{1}^{2}+x_{2}^{2}-5 x_{1} x_{2}\right)
\end{array}
$$

$$
\begin{array}{ll} 
& +w_{3}\left(5-\left(x_{1}-1\right)^{2}-\left(x_{2}-3\right)^{2}\right) \\
\text { s.t. } & x_{1}+x_{2}-8 \leq 0 \\
& x_{1}-x_{2}-2 \leq 0 \\
& x_{1}+x_{2}-4 \geq 0 \\
& x_{1}-x_{2}+2 \geq 0 \\
& f_{r}(x)=\bar{f}_{r}^{*},
\end{array}
$$

where $r \in\{1,2,3\}$.

$P 2$ :

$$
\begin{array}{ll}
\max & w_{1}\left(\left(x_{1}-4\right)^{2}+\left(x_{2}-2\right)^{2}-2\right) \\
& +w_{2}\left(x_{1}^{2}+x_{2}^{2}-5 x_{1} x_{2}\right)+ \\
& w_{3}\left(5-\left(x_{1}-1\right)^{2}-\left(x_{2}-3\right)^{2}\right) \\
\text { s.t. } & x_{1}+x_{2}-8 \leq 0 \\
& x_{1}-x_{2}-2 \leq 0 \\
& x_{1}+x_{2}-4 \geq 0 \\
& x_{1}-x_{2}+2 \geq 0 \\
& f_{r}(x) \geq \bar{f}_{r_{*}},
\end{array}
$$

where $r \in\{1,2,3\}$.

P3 :

$$
\begin{array}{ll}
\max & w_{1}\left(\left(x_{1}-4\right)^{2}+\left(x_{2}-2\right)^{2}-2\right) \\
& +w_{2}\left(x_{1}^{2}+x_{2}^{2}-5 x_{1} x_{2}\right)+ \\
& w_{3}\left(5-\left(x_{1}-1\right)^{2}-\left(x_{2}-3\right)^{2}\right) \\
\text { s.t. } & \left(x_{1}-3\right)^{2}+\left(x_{2}-3\right)^{2} \leq 9 \\
& f_{r}(x)=\bar{f}_{r}{ }^{*},
\end{array}
$$

where $r \in\{1,2,3\}$.

$P 4$ :

$$
\begin{array}{ll}
\max & w_{1}\left(\left(x_{1}-4\right)^{2}+\left(x_{2}-2\right)^{2}-2\right) \\
& +w_{2}\left(x_{1}^{2}+x_{2}^{2}-5 x_{1} x_{2}\right)+ \\
& w_{3}\left(5-\left(x_{1}-1\right)^{2}-\left(x_{2}-3\right)^{2}\right) \\
\text { s.t. } & \left(x_{1}-3\right)^{2}+\left(x_{2}-3\right)^{2} \leq 9 \\
& f_{r}(x) \geq \bar{f}_{r_{*}},
\end{array}
$$


where $r \in\{1,2,3\}$.

By changing the weights, we can get different efficient solutions, which are handled by the DM (see Tables 1-4).

In Table 1 , since there is no $\hat{x} \in M_{*}$ such that

Table 1. Surely-feasible, and surely-efficient solutions with different weights to Problem $P 1$.

\begin{tabular}{cccccc}
\hline$r$ & $w_{1}$ & $w_{2}$ & $w_{3}$ & $x_{1}{ }^{*}$ & $x_{2}{ }^{*}$ \\
\hline 3 & 0.33 & 0.33 & 0.34 & 1.000606 & 2.999394 \\
3 & 0.1 & 0.1 & 0.8 & 1.000577 & 2.999423 \\
3 & 0.5 & 0.4 & 0.1 & 1.000580 & 2.99910 \\
\hline
\end{tabular}

Table 2. Surely-feasible and possibly-efficient solutions with different weights to Problem $P 2$.

\begin{tabular}{cccccc}
\hline$r$ & $w_{1}$ & $w_{2}$ & $w_{3}$ & $x_{1}{ }^{*}$ & $x_{2}{ }^{*}$ \\
\hline 1 & 0.33 & 0.33 & 0.34 & 1.000606 & 2.999394 \\
1 & 0.9 & 0.05 & 0.05 & 1 & 3 \\
1 & 0.2 & 0.3 & 0.5 & 1.000712 & 2.999298 \\
2 & 0.1 & 0.8 & 0.1 & 1 & 3 \\
2 & 0.2 & 0.4 & 0.4 & 0.999320 & 2.999850 \\
2 & 0.5 & 0.3 & 0.2 & 0.999352 & 3.000120 \\
3 & 0.33 & 0.33 & 0.34 & 1 & 3 \\
3 & 0.2 & 0.4 & 0.4 & 1 & 3 \\
3 & 0.5 & 0.4 & 0.1 & 1 & 3 \\
\hline
\end{tabular}

Table 3. Possibly-feasible and surely-efficient solutions with different weights to Problem P3.

\begin{tabular}{cccccc}
\hline$r$ & $w_{1}$ & $w_{2}$ & $w_{3}$ & $x_{1}{ }^{*}$ & $x_{2}{ }^{*}$ \\
\hline 1 & 0.33 & 0.33 & 0.34 & 0.8786796 & 5.121320 \\
1 & 0.8 & 0.1 & 0.1 & 0.8691861 & 5.111786 \\
1 & 0.4 & 0.5 & 0.1 & 0.8614629 & 5.103963 \\
2 & 0.4 & 0.5 & 0.1 & 4.019090 & 0.1783941 \\
2 & 0.7 & 0.2 & 0.1 & 4.019602 & 0.1785793 \\
2 & 0.1 & 0.3 & 0.6 & 4.019006 & 0.1783636 \\
3 & 0.33 & 0.33 & 0.34 & 0.9995549 & 2.999893 \\
3 & 0.2 & 0.4 & 0.4 & 0.9990667 & 3.000117 \\
3 & 0.5 & 0.4 & 0.1 & 0.9992445 & 2.999690 \\
\hline
\end{tabular}

Table 4. Possibly-feasible and possibly-efficient solutions with different weights to Problem $P 4$.

\begin{tabular}{cccccc}
\hline$r$ & $w_{1}$ & $w_{2}$ & $w_{3}$ & $x_{1}{ }^{*}$ & $x_{2}{ }^{*}$ \\
\hline 1 & 0.33 & 0.33 & 0.34 & 0.1724377 & 4.002443 \\
1 & 0.2 & 0.4 & 0.4 & 0.1534008 & 3.947034 \\
1 & 0.5 & 0.4 & 0.1 & 0.2214822 & 4.131300 \\
2 & 0.33 & 0.33 & 0.34 & 0.1724377 & 4.002443 \\
2 & 0.2 & 0.4 & 0.4 & 0.1534008 & 3.947034 \\
2 & 0.1 & 0.3 & 0.6 & 0.1119307 & 3.811823 \\
3 & 0.33 & 0.33 & 0.34 & 0.9996620 & 3.000140 \\
3 & 0.2 & 0.4 & 0.4 & 0.9995774 & 2.999938 \\
3 & 0.5 & 0.4 & 0.1 & 0.9996227 & 2.999917 \\
\hline
\end{tabular}

$f_{r}(\hat{x})=\bar{f}_{r}^{*}$ for $r=1,2$, the results are provided only for $r=3$.

Table 2, for all $r=1,2,3$, gives the point $x^{*}=$ $(1,3)$ as a surely-feasible and possibly-efficient solution. Table 3 presents different points of the set $S$ which are possibly-feasible and surely-efficient solutions. Finally, in Table 4, all of the obtained solutions are possiblyfeasible and possibly-efficient, which belong to the set $T$.

Example 3.3. Consider Example 3.2 where the third objective function is changed as follows:

$$
\max f_{3}(x)=2 x_{1}^{3} x_{2}-2 x_{1} x_{2}^{3} .
$$

Then, $\bar{f}_{3 *}=a_{3}=f_{3}(5,3)=480, \bar{f}_{3}^{*}=c_{3}=$ $f_{3}(5.99,3.2445)=985.4736$, and the rough optimal sets for the function $f_{3}$ are:

$$
\begin{aligned}
F O_{s s}^{3} & =\left\{x \in M_{*} \mid f_{3}(x)=\bar{f}_{3}^{*}=985.4736\right\}=\emptyset \\
F O_{s p}^{3} & =\left\{x \in M_{*} \mid f_{3}(x) \geq \bar{f}_{3 *}=480\right\}=\{(5,3)\} \\
F O_{p s}^{3} & =\left\{x \in M^{*} \mid f_{3}(x)=\bar{f}_{3}^{*}=985.4736\right\} \\
& =\{(5.99,3.2445)\} \\
F O_{p p}^{3} & =\left\{x \in M^{*} \mid f_{3}(x) \geq \bar{f}_{3 *}=480\right\} \\
& =\left\{\left(x_{1}, x_{2}\right) \in U: 2 x_{1}^{3} x_{2}-2 x_{1} x_{2}^{3} \geq 480\right\} .
\end{aligned}
$$

Hence, the rough completely optimal sets are:

$$
\begin{aligned}
F O_{s(s c)}= & F O_{s(p c)}=F O_{p(s c)}=\emptyset \\
F O_{p(p c)}= & \left\{x \in U:\left\{x_{1}-4\right)^{2}+\left(x_{2}-2\right)^{2} \geq 10\right\} \\
& \cap\left\{x_{1}^{2}+x_{2}^{2}-5 x_{1} x_{2} \geq-5\right\} \\
& \left.\cap\left\{2 x_{1}^{3} x_{2}-2 x_{1} x_{2}^{3} \geq 480\right\}\right\} .
\end{aligned}
$$

So, Problem (11) with the new objective function $f_{3}$ has neither surely-complete optimal solution nor surely-feasible and possibly-complete optimal solution.

At the last step, for obtaining the rough efficient solutions sets we need to find the sets $P, Q, S$, and $T$ as follows:

$$
\begin{aligned}
& P=\bigcup_{j=1}^{3} F O_{s s}^{j}=\emptyset, \\
& Q=\bigcup_{j=1}^{3} F O_{s p}^{j}=\{(1,3),(3,5),(3,1),(5,3)\},
\end{aligned}
$$




$$
\begin{aligned}
S= & \bigcup_{j=1}^{3} F O_{p s}^{j}=\{(0.8787,5.1213),(4.0209,0.1791), \\
& (5.99,3.2445)\}, \\
T= & \bigcup_{j=1}^{3} F O_{p p}^{j}=\left\{x \in U:\left\{\left(x_{1}-4\right)^{2}+\left(x_{2}-2\right)^{2} \geq 10\right\}\right. \\
& \cup\left\{x_{1}^{2}+x_{2}^{2}-5 x_{1} x_{2} \geq-5\right\} \\
& \left.\cup\left\{2 x_{1}^{3} x_{2}-2 x_{1} x_{2}^{3} \geq 480\right\}\right\} .
\end{aligned}
$$

$P=\emptyset$ means that there is no $\hat{x} \in M_{*}$ such that $f_{r}(\hat{x})=$ $\bar{f}_{r}^{*}$ for $r=1,2,3$. Thus, Problem $P 1$ (by replacement of the new objective function $f_{3}$ ) is infeasible and there is no surely-feasible and surely-efficient solution. For finding efficient solutions of other degrees, see Tables 5 , 6 , and 7 .

Example 3.4. Let $U=\left\{x=\left(x_{1}, x_{2}\right) \in R^{2} \mid-3 \leq\right.$ $\left.x_{1} \leq 3,-3 \leq x_{2} \leq 3\right\}$.

Suppose that $E$ is an equivalence relation on $U$ such that it generates a partition as $U / E=\left\{E_{i j} \mid\right.$

Table 5. Surely-feasible and possibly-efficient solutions with different weights to Problem P2.

\begin{tabular}{cccccc}
\hline$r$ & $w_{1}$ & $w_{2}$ & $w_{3}$ & $x_{1}{ }^{*}$ & $x_{2}{ }^{*}$ \\
\hline 1 & 0.33 & 0.33 & 0.34 & 1 & 3 \\
1 & 0.1 & 0.8 & 0.1 & 3 & 5 \\
1 & 0.7 & 0.1 & 0.2 & 1.000606 & 2.999394 \\
\hline 2 & 0.1 & 0.8 & 0.1 & 1 & 3 \\
2 & 0.2 & 0.4 & 0.4 & 1.00022 & 2.99911 \\
2 & 0.5 & 0.4 & 0.1 & 0.999105 & 3.000114 \\
\hline 3 & 0.33 & 0.33 & 0.34 & 5.000001 & 3.000001 \\
3 & 0.2 & 0.4 & 0.4 & 5 & 3 \\
3 & 0.5 & 0.4 & 0.1 & 5 & 2.999999 \\
\hline
\end{tabular}

Table 6. Possibly-feasible and surely-efficient solutions with different weights to Problem P3.

\begin{tabular}{cccccc}
\hline$r$ & $w_{1}$ & $w_{2}$ & $w_{3}$ & $x_{1}{ }^{*}$ & $x_{2}{ }^{*}$ \\
\hline 1 & 0.33 & 0.33 & 0.34 & 0.8786796 & 5.121320 \\
1 & 0.2 & 0.4 & 0.4 & 0.8786750 & 5.121532 \\
1 & 0.7 & 0.15 & 0.15 & 0.881485 & 5.100560 \\
\hline 2 & 0.33 & 0.33 & 0.34 & 4.023270 & 0.1799071 \\
2 & 0.2 & 0.4 & 0.4 & 4.023233 & 0.1798934 \\
2 & 0.5 & 0.4 & 0.1 & 4.023014 & 0.1798143 \\
\hline 3 & 0.33 & 0.33 & 0.34 & 5.990152 & 3.242885 \\
3 & 0.2 & 0.4 & 0.4 & 5.990023 & 3.244462 \\
3 & 0.7 & 0.15 & 0.15 & 5.990024 & 3.244446 \\
\hline
\end{tabular}

Table 7. Possibly-feasible and possibly-efficient solutions with different weights to Problem P4.

\begin{tabular}{cccccc}
\hline$r$ & $w_{1}$ & $w_{2}$ & $w_{3}$ & $x_{1}{ }^{*}$ & $x_{2}{ }^{*}$ \\
\hline 1 & 0.33 & 0.33 & 0.34 & 0.01112153 & 3.258080 \\
1 & 0.2 & 0.4 & 0.4 & 0.009590253 & 3.239686 \\
1 & 0.5 & 0.4 & 0.1 & 0.05469409 & 3.570240 \\
\hline 2 & 0.33 & 0.33 & 0.34 & 5.504623 & 1.348677 \\
2 & 0.2 & 0.4 & 0.4 & 5.504622 & 1.348678 \\
2 & 0.5 & 0.4 & 0.1 & 0.05469409 & 3.570240 \\
\hline 3 & 0.33 & 0.33 & 0.34 & 5.993313 & 3.200190 \\
3 & 0.2 & 0.4 & 0.4 & 5.993560 & 3.196473 \\
3 & 0.5 & 0.4 & 0.1 & 5.999507 & 3.054395 \\
\hline
\end{tabular}

$i, j=1, \ldots, 6\}$ where:

$$
\begin{aligned}
E_{i j}= & \left\{x \in U \mid j-1 \leq x_{1} \leq j-3,\right. \\
& \left.3-i \leq x_{2} \leq 4-i\right\} \text { for all } i, j=1, \ldots, 6 .
\end{aligned}
$$

Now, assume that $M$ is a rough feasible region in the approximation space $A=(U, E)$ such that the lower and upper approximations of $M$ are as follow:

$$
\begin{aligned}
& M_{*}=\left\{\left(x_{1}, x_{2}\right) \in U \mid-1 \leq x_{1} \leq 1, \quad-1 \leq x_{2} \leq 1\right\}, \\
& M^{*}=\left\{\left(x_{1}, x_{2}\right) \in U \mid-2 \leq x_{1} \leq 2, \quad-2 \leq x_{2} \leq 2\right\} .
\end{aligned}
$$

Also, the boundary region of $M$ is given by:

$$
\begin{aligned}
M_{B N}= & \left\{\left(x_{1}, x_{2}\right) \in U \mid-2 \leq x_{1}<-1,1<x_{1} \leq 2,\right. \\
& \left.-2 \leq x_{2}<-1,1<x_{2} \leq 2\right\},
\end{aligned}
$$

(see Figure 2). We consider the following RMOP problem:

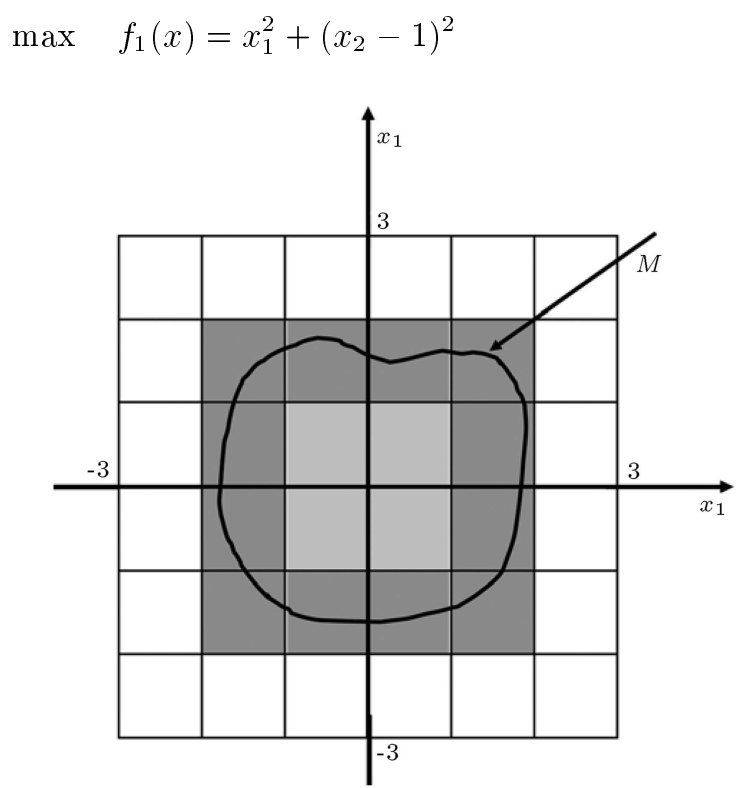

Figure 2. The rough set $M$ and its approximations. 


$$
\begin{array}{ll}
\max & f_{2}(x)=\sqrt{2 x_{1} x_{2}+5}-x_{2}^{3} \\
\max & f_{3}(x)=2 x_{1} x_{2}-x_{1}^{2} \\
\text { s.t. } & x \in M \\
& M_{*} \subseteq M \subseteq M^{*} .
\end{array}
$$

To find the solutions of Problem (12), similar to Example 3.2, we proceed as follow:

First step: Finding the rough optimal value $\bar{f}=$ $\left(\bar{f}_{1}, \bar{f}_{2}, \bar{f}_{3}\right)$ :

$$
\begin{aligned}
& \bar{f}_{1 *}=a_{1}=f_{1}(-1,-1)=f_{1}(1,-1)=5, \\
& \bar{f}_{1}^{*}=c_{1}=f_{1}(-2,-2)=f_{1}(2,-2)=13, \\
& \bar{f}_{2 *}=a_{2}=f_{2}(-1,-1)=\sqrt{7}+1, \\
& \bar{f}_{2}^{*}=c_{2}=f_{2}(-2,-2)=\sqrt{13}+8, \\
& \bar{f}_{3 *}=a_{3}=f_{3}(-1,-1)=f_{3}(-1,-1)=1, \\
& \bar{f}_{3}^{*}=c_{3}=f_{3}(2,2)=f_{3}(-2,-2)=4 .
\end{aligned}
$$

Second step: Finding the rough optimal sets for any objective function:

$$
\begin{aligned}
& F O_{s s}^{1}=\left\{x \in M_{*} \mid f_{1}(x)=\bar{f}_{1}^{*}=13\right\}=\emptyset, \\
& F O_{s p}^{1}=\left\{x \in M_{*} \mid f_{1}(x) \geq \bar{f}_{1 *}=5\right\} \\
& =\{(-1,-1),(1,-1)\}, \\
& F O_{p s}^{1}=\left\{x \in M^{*} \mid f_{1}(x)=\bar{f}_{1}^{*}=13\right\} \\
& =\{(-2,-2),(2,-2)\}, \\
& F O_{p p}^{1}=\left\{x \in M^{*} \mid f_{1}(x) \geq \bar{f}_{1 *}=5\right\} \\
& =\left\{\left(x_{1}, x_{2}\right) \in U: x_{1}^{2}+\left(x_{2}-1\right)^{2} \geq 5\right\}, \\
& F O_{s s}^{2}=\left\{x \in M_{*} \mid f_{2}(x)=\bar{f}_{2}^{*}=\sqrt{13}+8\right\}=\emptyset, \\
& F O_{s p}^{2}=\left\{x \in M_{*} \mid f_{2}(x) \geq \bar{f}_{2 *}=\sqrt{7}+1\right\} \\
& =\{(-1,-1)\}, \\
& F O_{p s}^{2}=\left\{x \in M^{*} \mid f_{2}(x)=\bar{f}_{2}^{*}=\sqrt{13}+8\right\} \\
& =\{(-2,-2)\}, \\
& F O_{p p}^{2}=\left\{x \in M^{*} \mid f_{2}(x) \geq \bar{f}_{2 *}\right\} \\
& =\left\{\left(x_{1}, x_{2}\right) \in U: \sqrt{2 x_{1} x_{2}+5}-x_{2}^{3} \geq \sqrt{7}+1\right\},
\end{aligned}
$$

$$
\begin{aligned}
F O_{s s}^{3} & =\left\{x \in M_{*} \mid f_{3}(x)=\bar{f}_{3}^{*}=4\right\}=\emptyset \\
F O_{s p}^{3} & =\left\{x \in M_{*} \mid f_{3}(x) \geq \bar{f}_{3 *}=1\right\} \\
& =\{(-1,-1),(1,1)\} \\
F O_{p s}^{3} & =\left\{x \in M^{*} \mid f_{3}(x)=\bar{f}_{3}^{*}=4\right\} \\
& =\{(-2,-2),(2,2)\} \\
F O_{p p}^{3} & =\left\{x \in M^{*} \mid f_{3}(x) \geq \bar{f}_{3 *}=1\right\} \\
& =\left\{\left(x_{1}, x_{2}\right) \in U: 2 x_{1} x_{2}-x_{1}^{2} \geq 1\right\} .
\end{aligned}
$$

Third step: Finding the rough completely optimal sets:

$$
\begin{aligned}
F O_{s(s c)}= & \bigcap_{j=1}^{3} F O_{s s}^{j}=\emptyset, \\
F O_{s(p c)}= & \bigcap_{j=1}^{3} F O_{s p}^{j}=\{(-1,-1)\}, \\
F O_{p(s c)}= & \bigcap_{j=1}^{3} F O_{p s}^{j}=\{(-2,-2)\}, \\
F O_{p(p c)}= & \left\{x \in U:\left\{x_{1}^{2}+\left(x_{2}-1\right)^{2} \geq 5\right\}\right. \\
& \cap\left\{\sqrt{2 x_{1} x_{2}+5}-x_{2}^{3} \geq \sqrt{7}+1\right\} \\
& \left.\cap\left\{2 x_{1} x_{2}-x_{1}^{2} \geq 1\right\}\right\} .
\end{aligned}
$$

Therefore, Problem (12) has no surely-feasible and surely-complete optimal solution. But, the point $x^{*}=$ $(-1,-1)$ is a surely-feasible and possibly-complete optimal solution and $\bar{x}=(-2,-2)$ is a possibly-feasible and surely-complete optimal solution.

Fourth step: Finding the rough efficient solutions set, firstly, the sets $P, Q, S$, and $T$ are as follow:

$$
\begin{aligned}
& P=\bigcup_{j=1}^{3} F O_{s s}^{j}=\emptyset, \\
& Q=\bigcup_{j=1}^{3} F O_{s p}^{j}=\{(-1,-1),(1,-1),(1,1)\}, \\
& S=\bigcup_{j=1}^{3} F O_{p s}^{j}=\{(-2,-2),(2,-2),(2,2)\}, \\
& T=\bigcup_{j=1}^{3} F O_{p p}^{j}=\left\{x \in U:\left\{x_{1}^{2}+\left(x_{2}-1\right)^{2} \geq 5\right\}\right.
\end{aligned}
$$




$$
\begin{aligned}
& \cup\left\{\sqrt{2 x_{1} x_{2}+5}-x_{2}^{3} \geq \sqrt{7}+1\right\} \\
& \left.\cup\left\{2 x_{1} x_{2}-x_{1}^{2} \geq 1\right\}\right\} .
\end{aligned}
$$

Secondly, according to Theorems $3.2-3.5$, we need to solve the SOP problems $Q 1, Q 2, Q 3$ and $Q 4$ with different weights.

$$
\text { Q1: }
$$

$$
\begin{array}{ll}
\max \quad w_{1} & \left(x_{1}^{2}+\left(x_{2}-1\right)^{2}\right) \\
& +w_{2}\left(\sqrt{2 x_{1} x_{2}+5}-x_{2}^{3}\right)+w_{3}\left(2 x_{1} x_{2}-x_{1}^{2}\right) \\
\text { s.t. } & -1 \leq x_{1} \leq 1 \\
& -1 \leq x_{2} \leq 1 \\
f_{r}(x) & =\bar{f}_{r}^{*},
\end{array}
$$

where $r \in\{1,2,3\}$.

$Q 2$ :

$$
\begin{array}{ll}
\max & w_{1}\left(x_{1}^{2}+\left(x_{2}-1\right)^{2}\right) \\
& +w_{2}\left(\sqrt{2 x_{1} x_{2}+5}-x_{2}^{3}\right)+w_{3}\left(2 x_{1} x_{2}-x_{1}^{2}\right) \\
\text { s.t. } & -1 \leq x_{1} \leq 1 \\
& -1 \leq x_{2} \leq 1 \\
& f_{r}(x) \geq \bar{f}_{r *},
\end{array}
$$

where $r \in\{1,2,3\}$.

Q3:

$$
\begin{array}{ll}
\max & w_{1}\left(x_{1}^{2}+\left(x_{2}-1\right)^{2}\right) \\
& +w_{2}\left(\sqrt{2 x_{1} x_{2}+5}-x_{2}^{3}\right)+w_{3}\left(2 x_{1} x_{2}-x_{1}^{2}\right) \\
\text { s.t. } & -2 \leq x_{1} \leq 2 \\
& -2 \leq x_{2} \leq 2 \\
& f_{r}(x)=\bar{f}_{r}^{*},
\end{array}
$$

where $r \in\{1,2,3\}$.

Q4:

$$
\begin{array}{ll}
\max & w_{1}\left(x_{1}^{2}+\left(x_{2}-1\right)^{2}\right) \\
& +w_{2}\left(\sqrt{2 x_{1} x_{2}+5}-x_{2}^{3}\right)+w_{3}\left(2 x_{1} x_{2}-x_{1}^{2}\right) \\
\text { s.t. } & -2 \leq x_{1} \leq 2 \\
& -2 \leq x_{2} \leq 2 \\
& f_{r}(x) \geq \bar{f}_{r *},
\end{array}
$$

where $r \in\{1,2,3\}$.

Therefore, we can get the rough efficient solutions sets of different degrees. Since $P=\emptyset$, there is no $\hat{x} \in M_{*}$ such that $f_{r}(\hat{x})=\bar{f}_{r}^{*}$ for $r=1,2,3$. Thus, Problem $Q 1$ is infeasible and there is no surely-feasible and surely-efficient solution. But, for finding efficient solutions of other degrees, the rest of calculations are similar to those of Examples 3.2 and 3.3.

Remark 3.3. Note that it is possible that some of the sets $P, Q, S$, and $T$ be empty. In this case, further research is needed to find surely- and possibly-efficient solutions to RMOP problems.

\section{Conclusion}

In this paper, we first reviewed the basic concepts of rough set theory and recalled some preliminary concepts and results of the kinds of solutions of a multiobjective programming problem by paying special attention to a scalarization method used to obtain a solution. We also reviewed rough programming problems and introduced the concept of Rough Multi-Objective Programming (RMOP) problems. Then RMOP problems were classified into five classes. We have just proposed a method for obtaining the solutions of the first class, where the feasible region is a rough set, while all the objective functions are crisp. In this regard, we discussed new concepts such as "surely-complete optimal solutions", "possibly-complete optimal solutions", "surely-efficient solutions" and "possibly-efficient solutions". Also, we solved some numerical examples for illustration.

Extending our study to the remaining four other classes is a subject for future research. Application of our methods to real-world problems may also lead to further considerations.

\section{Acknowledgments}

The authors would like to express their gratitude to the anonymous reviewers for the comments and suggestions, which greatly helped them to improve the paper.

\section{References}

1. Ehrgott, M., Multicriteria Optimization, 2nd Ed., Springer, Berlin (2005).

2. Pawlak, Z. "Rough sets", International Journal of Information and Computer Sciences, 11(5), pp. 341356 (1982).

3. Liu, B. "Theory and practice of uncertain programming", Physica Verlag, New York (2002).

4. Xu, J. and Tao, Z., Rough Multiple Objective Decision Making, CRC Press, New York (2011). 
5. Hamzehee, A., Yaghoobi, M.A. and Mashinchi, M. "Linear programming with rough interval coefficients", Journal of Intelligent and Fuzzy Systems, 26, pp. 11791189 (2014).

6. Youness, E.A. "Characterizing solutions of rough programming problems", European Journal of Operational Research, 168(3), pp. 1019-1029 (2006).

7. Osman, M.S., Lashein, E.F., Youness, E.A. and Atteya, T.E.M. "Mathematical programming in rough environment", Optimization, 60(5), pp. 603-611 (2011).

8. Sakawa, M. "Fuzzy sets and interactive multiobjective optimization", Plenum Press, New York and London (1993).

9. Greco, S., Matarazzo, B. and Slowinski, R. "Rough set theory for multicriteria decision analysis", European Journal of Operational Research, 129(1), pp. 1-47 (2001).

10. $\mathrm{Xu}, \mathrm{J}$. and Zhao, L. "A class of fuzzy rough expected value multi-objective decision making model and its application to inventory problems", Computers and Mathematics with Applications, 56(8), pp. 2107-2119 (2008).

11. $\mathrm{Xu}$, J. and Zhao, L. "A multi-objective decisionmaking model with fuzzy rough coefficients and its application to the inventory problem", Information Sciences, 180(5), pp. 679-696 (2010).

12. Xu, J. and Yao, L. "A class of multiobjective linear programming models with random rough coefficients", Mathematical and Computer Modelling, 49(1-2), pp. 189-206 (2009).

13. Xu, J. and Yao, L. "A class of expected value multiobjective programming problems with random rough coefficients", Mathematical and Computer Modelling, 50(1-2), pp. 141-158 (2009).

14. Xu, J., Li, B. and Wu, D. "Rough data envelopment analysis and its application to supply chain performance evaluation", International Journal of Production Economics, 122(2), pp. 628-638 (2009).

15. Tao, Z. and Xu, J. "A class of rough multiple objective programming and its application to solid transportation problem", Information Sciences, 188, pp. 215-235 (2012).

16. Li, F., Jing, Y., Shi, Y. and Jin, C. "Research on the theory and method of rough programming based on synthesis effect", International Journal of Innovative Computing, Information and Control, 9(5), pp. 20872098 (2013).
17. Li, F., Jin, C., Jing, Y., Wilamowska-Korsak, M. and $\mathrm{Bi}, \mathrm{Z}$. "A rough programming model based on the greatest compatible classes and synthesis effect", Systems Research and Behavioral Science, 30(3), pp. 229-243 (2013).

18. Pawlak, Z. "Rough sets, rough relations and rough functions", Fundamental Informaticae, 27, pp. 103-108 (1996).

\section{Biographies}

Ali Hamzehee was born in 1974 in Iran. He received his BS and MS degrees both in Applied Mathematics from Vali Asr University of Rafsanjan and Amir Kabir University of Tehran, in 1997 and 1999, respectively. $\mathrm{He}$ is currently a PhD student in Department of Mathematics, Faculty of Mathematics and Computer, Shahid Bahonar University of Kerman. Currently, he is a Lecturer at the Department of Mathematics in Islamic Azad University, Kerman Branch. His research interests include operational research, decision making, fuzzy programming and rough programming.

Mohammad Ali Yaghoobi is Assistant Professor in the Mathematics Department at the Shahid Bahonar University of Kerman (SBUK), Iran, where he was the Head of Statistics Department from December 2006 to March 2009. He received his MS degree, ranked first, in Applied Mathematics and his $\mathrm{PhD}$ degree in the same field in branch of Operational Research from the SBUK. His main research interests are in the fields of multiple criteria decision making, goal programming, fractional programming, linear and integer programming, and fuzzy programming. He has published several papers in national and international journals.

Mashaallah Mashinchi was born in 1951 in Iran. He received his BS and MS degrees both in Statistics, from Ferdowsi University of Mashhad and Shiraz University, in 1976 and 1978, respectively, and his $\mathrm{PhD}$ degree in Mathematics from Waseda University in Japan, in 1987. He is now a Professor in the Department of Statistics at Shahid Bahonar University of Kerman (SBUK), Iran. He is also Editor-in-Chief of the Iranian Journal of Fuzzy Systems, and the Director of the Fuzzy Systems \& Applications Center of Excellence at SUBK. His current research interests include fuzzy mathematics, especially in statistics, decision making and algebraic systems. 